\title{
Experimentelle Beiträge zur Frage der Mesothoriumschädigung des Darmes.
}

Von

\author{
0. Pankow (Düsseldorf).
}

(Hernu Tafel XI und 4 Textfiguren.)

Als man nach der Aufnahme der vaginalen Mesothorium- und Radiumbehandlung des Uteruskarzinoms die anfangs nicht gerade seltenen Darmschädigungen und Mastdarm-Scheidenlisteln beobachtete, wollte man ihre Entstehung zunächst nicht aussehliesslich der damals noch vielfach üblichen Methode zur Last legen, die mit raschen und häufigen Wiederholungen der Bestrahlungen bei verhältnismässig grossen Dosen und geringem Abstand arbeitete. Man suchte vielmehr nach begünstigenden Momenten. So wollte man die Verbrennungen und Fistelbildungen z. B. mit einem undichten Filter erklären, eine Ansicht, die aber von vornherein nicht anerkannt wurde. Dann glaubte man vor allem, dass in Fällen derartiger Fistelbildung bereits ein wenn auch makroskopisch noch nicht sichtbares Uebergreifen des Karzinoms auf die Scheide stattgefunden hätte. Schon bald nachdem wir die Radium- und Mesothoriumbehandlung in unserer Klinik aufgenommen hatten, sahen wir jedoch bei einer Patientin mit ganz beginnendem Karzinom, bei der wegen schweren Herzfehlers ein operativer Eingriff nicht in Frage kam, eine solche Fistel entstehen. Dem ganzen Befunde nach konnte man eine weitgehende subepitheliale Ausbreitung des Karzinoms auf die Vagina als begünstigendes Moment für die Entstehung dieser Fistel nicht annehmen. Ich liess deshalb durch den Oberarzt der Klinik, Dr. Borell, experimentelle Untersuchungen über diese Frage anstellen. Ganz kurz habe ich darüber bereits in der Sitzung der Niederrheinisch-Westfälischen Gesellschaft für Geburtshilfe und Gynäkologie im Mai 1914 berichtet. Von einer Veröffentlichung der Resultate wurde damals abgesehen, da wir nach den interessanten Ergebnissen am Mastdarme die Bestrahlungs- 
wirkung auch noch an anderen Darmabschnitten studieren wollten. Da wir dazu grössere Strecken des Darmes an der Bauchwand fixieren mussten, um sie unverschieblich und der angewandten Mesothoriummenge ganz gleichmässig zugänglich zu halten, ein Teil der Versuchstiere dann aber sehr bald zugrunde ging, hatten wir das Material noch nicht so völlig zusammen, wie es nötig gewesen wäre, als der Krieg ausbrach. Da auch vorerst an einen Fortgang dieser Versuche nicht zu denken ist, möchte ich jetzt nur die wichtigsten Ergebnisse der Experimente schildern, die sich auf die Schädigungen des Mastdarms beziehen.

Die Bestrahlung wurde in der Weise ausgeführt, dass $50 \mathrm{mg}$ Nesothorium, in $1 \mathrm{~mm}$ Blei und mit $1 \mathrm{~mm}$ Paragummi überzogen, in die Scheide des Kaninchens eingeschoben wurde.

Um ein Herausgleiten zu verhüten, wurde die Vagina durch eine Knopfnaht geschlossen. In Pausen von 8 Tagen wurde diese Bestrahlung mit einer Dauer von 10-24 Stunden wiederholt.

Es zeigte sich hierbei, dass das Liegen der Kapseln auch auf lange Stunden hin von den Kaninchen ohne merkbare Beschwerden ertragen wurde; erst nach wiederholten Bestrahlungen traten bei den Tieren die ersten klinischen Erscheinungen ein, die im Abgang blutig gefärbter Ausleerungen bestanden. Das gleicht durchaus den Beobachtungen am Menschen. Wir betrachten bei unserem klinischen Material dieses Symptom, auch wenn sonst keinerlei ernstere Störungen bestehen und durch die Palpation und Inspektion keine Veränderungen als Folge der Bestrahlungen nachzuweisen sind, doch als eine ganz besondere Mahnung zur Vorsicht bei der weiteren vaginalen Behandlung, zumal uns die Rektoskopie solcher Fälle gezeigt hat, dass sich auch selbst schon im Beginn dieser Erscheinungen recht dentliche Veränderungen im Darme nachweisen lassen.

Fran K., 56 Jahre alt, Carcinoma cervicis inoperabile. Der Mastdarm bei Beginn der Behandlung vollständig unverändert.

Nach dreimaliger vaginaler Bestrahlung in Pausen von 8 Tagen mit je $100 \mathrm{mg}$ Mesothorium in $2 \mathrm{~mm}$ Blei, $1 \mathrm{~mm}$ Paragummi und $1 \mathrm{~cm}$ Gaze für je 24 Stunden klagt die Patientin über Drang zum Stuhlgang und Schmerzen bei den Entleerungen, die, wie sie angibt, in der letzten Zeit oft blutig gefärbt sind und häufig „wässrig“ aussehen. Die rektoskopische Untersuchung ergab: Die ganze Rektalschleimhaut ist mit. blutigen, schleimigen Massen bedeckt, die an der Vorderwand des Rektums fester haften als an der Hinterwand; hier blutet es auch beim Abtupfen der Auflagerungen diffus, und die ganze Mukosa ist unter der Schleimhautschicht stark fleckig gerötet. Auch an der Hinterwand und an den Seitenwänden finden sich diese schleimigen Auflagerungen; nach 
dem Abtupfen ist hier aber das Gesamtaussehen weniger stark verändert als an der Vorderwand. Ein ausgesprochenes Ulkus findet sich nicht im Rektum.

Ganz ähnlich wie der klinische Befund dieser Patientin, die auffallend früh klinische Reaktionen zeigte - wie denn überbaupt die Reaktion bei gleicher Dosierung subjektiv überaus verschieden ist -, war der Befund bei einem Tiere, dessen Mastdarm den geringsten Grad der Schädigung zeigte.

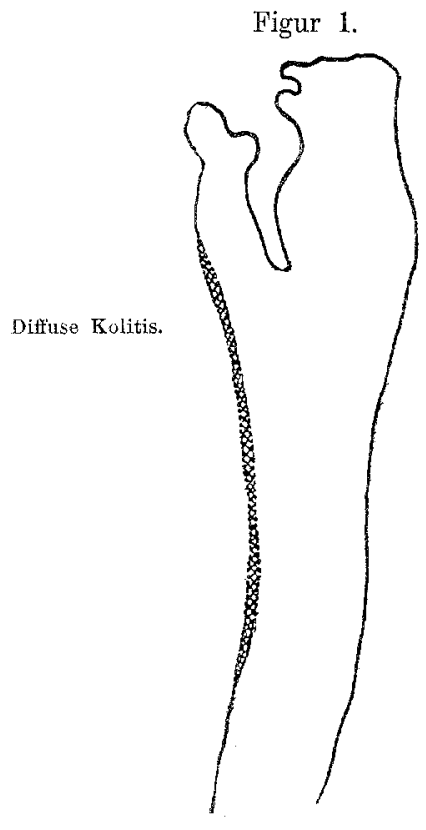

Kaninchen I.

Fall I (Fig. 1). Dieses Tier, das in der Zeit vom 9. II. bis 21. III. fünfmal bestrahlt war, abortierte am 23. UII. und starb. Der Befund war folgender: Abgesehen von der Umgebung der Nahtstelle findet sich in der Vagina keinerlei Veränderung, und das Oberflächenepithel ist überall erhalten. Im Darme sieht man eine flache, stark infiltrierte Schleimhaut. Die Drüsenschlänche sind zum Teil zugrunde gegangen und grösstenteils in blasse, homogene Gebilde umgewandelt, in denen die Kerne mangelhaft gefärbt sind. Das oberfächliche Zylinderepithel ist völlig intakt. Eine auf der Abbildung (s. Taf XI, Fig. 1) von der Seite her herüberliegende und flach getroffene Schleimhautfalte lässt die Verhältnisse besonders gut erkennen. Die Darmmuskulatur ist überall gut erhalten und weist spärliche Leukozyteneinlagerungen auf. Die Submukosa ist dagegen dicht mit Leukozyten durchsetzt. Das Bindegewebe zwischen Darm und Scheide zeigt teilweise starke hyaline Degeneration. Um die Gefässe herum sind auch hier kleine Leukozytenherde festzustellen. 
Hier zeigt sich also eine weitgehende Uebereinstimmung des klinischen Befundes beim Menschen mit dem Obduktionsbefunde beim Kaninchen. Liegt einmal ein solcher klinischer Befund beim Mensehen vor, dann pflegt besonders bei noch weiter wiederholten Bestrahlungen der Prozess weiter zu gehen, und auf solcher Basis entstehen dann die Ulzerationen, die schliesslich zur Fistelbildung führen. Genau so ist es aber auch beim Kaninchen.

Figur 2.



Kaninchen II.

Tier II, Fall II (Fig. 2), das in gleicher Weise fünfmal bestrahlt war wie Tier I, zeigte die gleichen klinischen Erscheinungen wie dieses. Es starb nicht, nahm aber nach der letzten Bestrahlung bei herabgesetzter Nahrungsaufnahme sichtlich ab und wurde 20 Tage nach der Ietzten Behandlung getötet. Der Befund war folgender (Taf. XI, Fig. 2): Makroskopisch ist die Scheide vollkommen unverändert; im Rektum dagegen sieht man ein kleines Geschwür von etwa $1 \mathrm{~cm}$ Länge. Es beginnt an der Stelle, wo das Plattenepithel des Anus in das Zylinderepithel der Darmschleimhaut übergeht; an der tiefsten Partie greift es bis auf den Boden der glatten Darmmuskulatur über. Die Schleimhaut und die Muskulatur sind im Gebiete des Geschwürs vollkommen nekrotisch. Weiter oben schliesst sich dann eine Zone an, in der die Darmschleimhaut Veränderungen zeigt, wie sie beim Tier I beschrieben sind, während die Muskulatur fast völlig unverändert aussieht. An Stelle der Drüsen sieht man helle homogene Gebilde, in denen die unregelmässig gelagerten blassen Kerne undeutlich zu erkennen sind. 


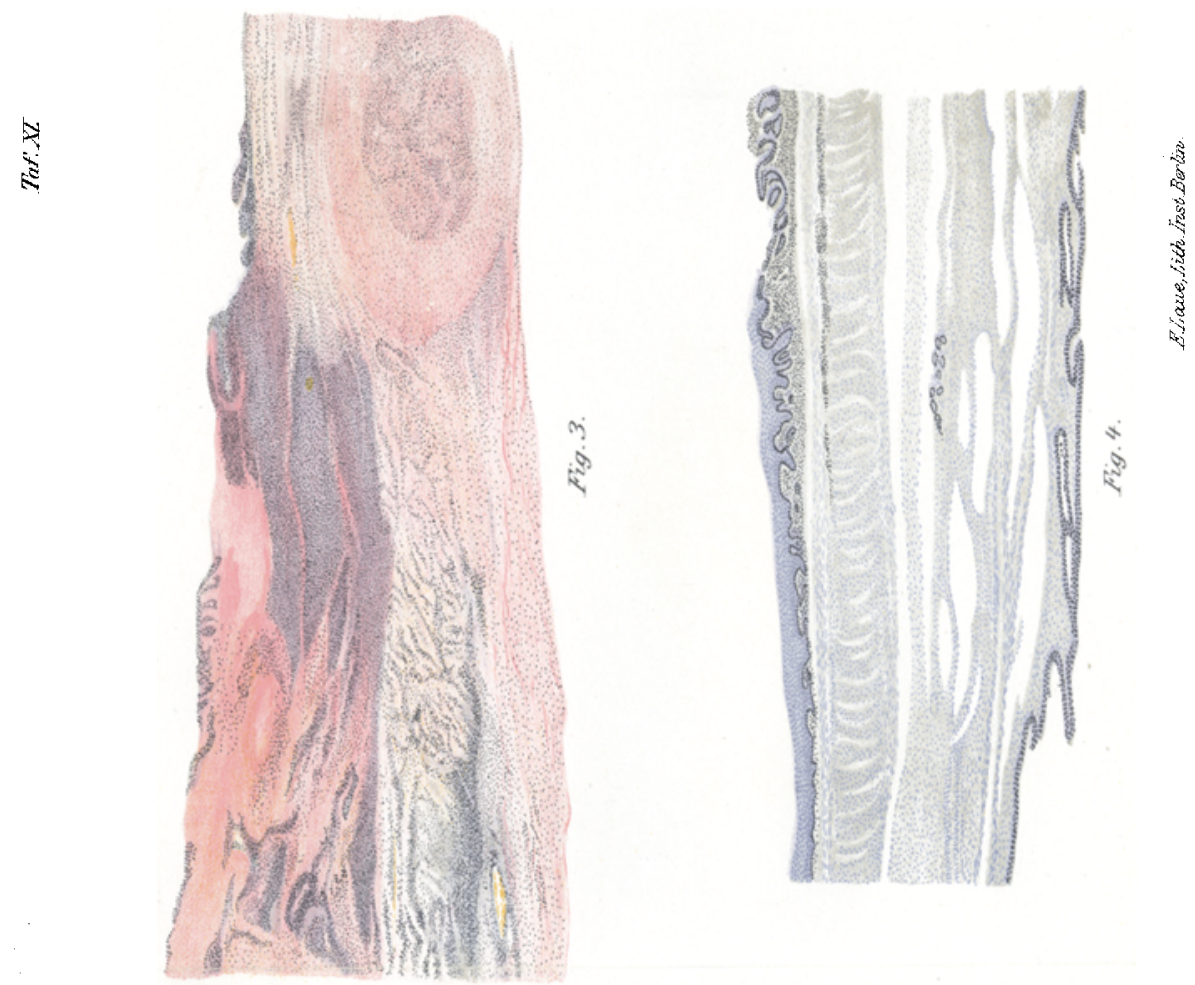

है
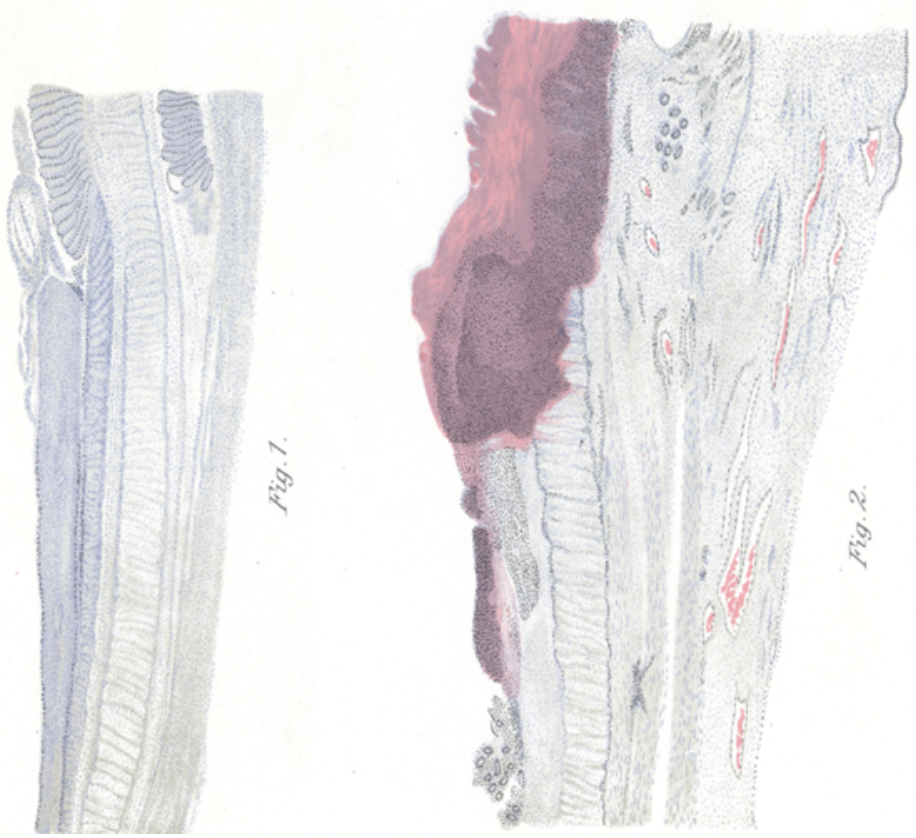
Zwischen diesen Gebilden liegen unzählige Leukozyten. Allmählich setzen dann in grösserer Entfernung von dem Geschwür normale Drüsen ein, und die Leukozyteninfiltration nimmt ab. Rings um die nekrotischen Massen liegen auch in der Muskulatur sehr zahlreiche Leukozyten. Im übrigen finden sich an den erhaltenen Muskelzellen keine nachweisbaren Schädigungen. Thre Grösse und Form ist normal, der Kern ist gut darstellbar. Hingegen weist das zwischen Darm und Scheide gelegene Bindegewebe gewisse Veränderungen auf; es ist homogen, fast völlig strukturlos, und wenn sich Kerne zeigen, so färben sie sich nur sehr matt. Auch hier wie in der Muskulatur des Darmes finden sich verstreut uiberall Leukozyten. Um die kleineren Gefässe sind sie zu dichten Gruppen angeordnet. Die Scheidenschleimhaut ist völlig normal.

Während bei diesen beiden Versuchen das Vaginalrohr aber noch keine Veränderungen aufwies, zeigte sich bei den weiteren Versuchen, bei denen wir statt der 10 stündigen Behandlung eine 24 stündige gewählt hatten, genau so wie beim Menschen nun auch bei den Kaninchen eine ausgedehnte Geschwürsbildung im Rektum, zugleich aber auch eine, wenn auch kleinere, Gesehwürsbildung in der Vagina, die bei längerem Fortbestehen wiederum ebenso wie beim Menschen zu einer ausgesprochenen Stenose beider Röhren führte. Diese Zustände sind ja beim Menschen oft mit ganz unerträglichen Schmerzen verbunden. Auch beim Tier äussern sich die Erscheinungen in ganz gleicher Weise. Die Kaninchen magerten bei solchen Befunden zusehends ab, frassen schlecht und liessen in den letzten Tagen so lebhafte Schmerzäusserungen hören, dass sie früher getötet werden mussten, als eigentlich beabsichtigt war.

Den geringeren Grad einer solchen Veränderung zeigt die folgende Beobachtung:

Tier III (Fig. 3): Schon makroskopisch kann man sowohl am Darme wie auch an der gegenüberliegenden Vaginalschleimhaut eine im Rektum jedoch tiefergreifende Ulzeration erkennen. Es ist indessen zwischen beiden Geschwüren noch eine intakte Gewebsschicht vorhanden; auch besteht insofern ein Unterschied, als die Ausdehnung des Darmgeschwürs überhaupt erheblich grösser ist als die des Vaginalulkus. Mikroskopisch findet man die Schleimhaut des Darmes in einer Ausdehnung von $2 \mathrm{~cm}$ schwer geschädigt. Das Ulkus greift bis auf den Boden der Darmmuskulatur über, die ebenso wie die Schleimhaut vollkommen nekrotisch ist und eine völlig verwaschene Zeichnung aufweist (Taf. XI, Fig. 3). Vielfach kann man jedoch noch die ursprüngliche Struktur der Muskulatur erkennen. Die Nekrose ist abgeschlossen durch einen Leukozytenwall. Zu beiden Seiten des Ulkus erholt sich am ehesten wieder die Muskulatur, während die Schleimhaut, die noch weithin mit Lenkozyten durchsetzt ist, anstatt der Drüsen nur noch homogene Schläuche mit Drüsenkernen aufweist. In einer Entfernung 
von $1 \mathrm{~cm}$ von dem eigentlichen Geschwür sind noch keine normalen Drüsen zu finden. Stärker als bei Kaninchen II ist die Infiltration des Zwischengewebes mit Leukozyten und die hyaline Degeneration.

Das etwa $1 / 2 . \mathrm{cm}$ lange und $3 \mathrm{~mm}$ tiefe Ulkus der Vagina liegt ziemlich nahe am Scheideneingange. Hier zeigt das Zwischengewebe zwischen Darm und Scheide überall eine starke Leukozyteninfiltration und weist in seinen bindegewebigen Bestandteilen eine hyaline Degeneration auf. Im übrigen ist die Scheidewand noch ziemlich dick, und nur diesem Umstande ist es wohl zu danken, dass sich keine Fistel gebildet hat. Das Ulkus ist von zahlreichen Leukozyten umgeben. Das

Figur 3.

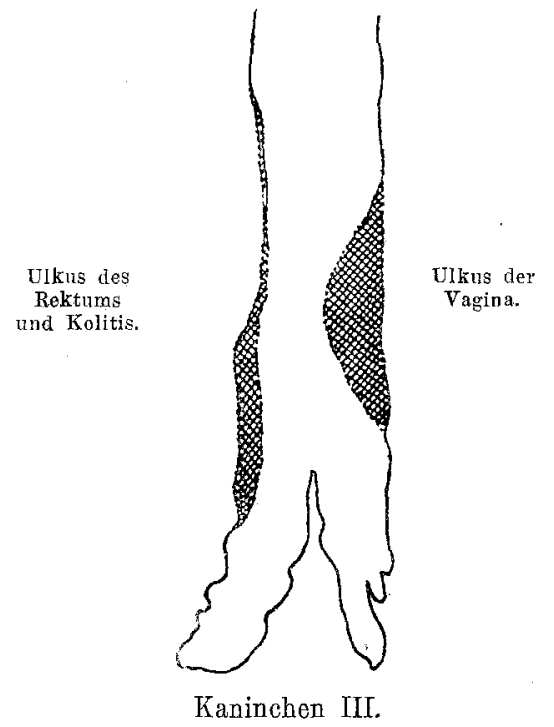

Plattenepithel oberhalb und unterhalb des Ulkus ist normal; es besteht also hier im Gegensatz zum Rektumulkus ein sehr scharfer Uebergang von der gesunden zur kranken Schleimhaut. Beide Rohre sind im Gebiete des Ulkus sehr stark verengert.

Hält man sich die hier wiedergegebenen Befunde vor Augen, so sieht man daran die ganz auffallende Uebereinstimmung mit den Befunden beim Menschen. Hier wie dort treten zunächst klinische Erscheinungen seitens des Darmes auf, die in Durchfällen, blutig-wässrigen Stühlen, bestehen und sich zu Geschwürsbildungen und hochgradiger Stenose mit intensiven Schmerzen steigern. Ich zweille nicht, dass, wenn man Tiere, die wie das letzte bestrahlt sind, noch längere Zeit am Leben lassen würde, man dann genau wie beim Menschen auch eine ausgesprochene Fistelbildung erleben würde. Ist erst die Nekrose so tief gegangen wie bei dem letzten Tier, und kommen dann neben den fort- 
schreitenden Bestrahlungssehädigungen anch noch die Wirkungen der Kotinfektion hinzu, dann ist es verständlich, dass Fisteln entstehen müssen. Ich habe gerade bei den Versuchen am Kaninchen den Eindruck gewonnen, dass die Fistelbildungen sehliesslich durch Vertiefung der Ulzeration vom Rektum her entstehen.

Wie sich die Verhältnisse gestalten würden, wenn man beim Eintritt der ersten anatomischen Erscheinungen mit der Bestrahlung

Figur 4.

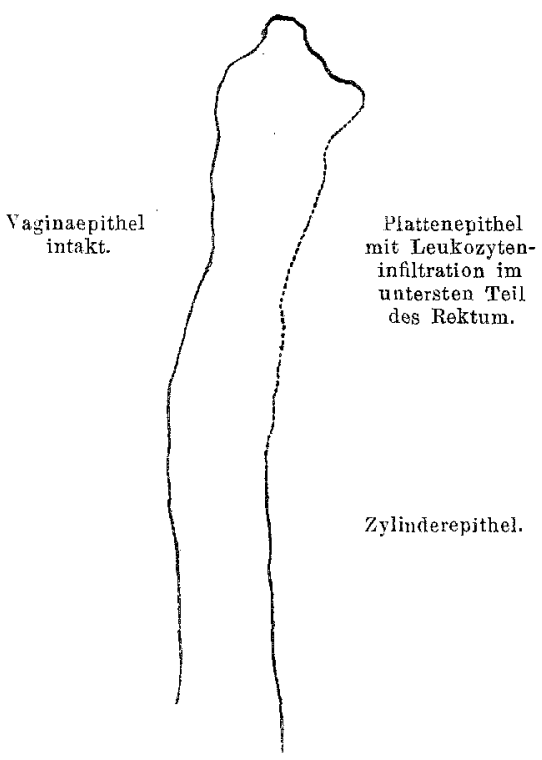

Kaninchen IV.

aufhören würde, darüber möchte ich mich nicht aussprechen. Anfangs glaubte ich, dass bei Fällen von Kolitis und leichter Geschwürsbildung durch Hineinwuchern des Plattenepithels des Anus in das Rektum eine Ausheilung dieser Veränderungen stattfinden könne (Fig. 4).

Das in Taf. XI Fig. 4 wiedergegebene Bild stelit den Befund bei einem Tiere dar, das ebenso wie Tier I innerhalb 39 Tagen fünfmal je 10 Minuten bestrahlt war, dann aber noch 8 Wochen am Leben blieb und nun erst getötet wurde. Makroskopisch sieht die Vagina völlig unverändert aus. Bei der mikroskopischen Untersuchung zeigte sich folgendes: In der Vaginalwand finden sich ausserordentlich weite Lymphspalten, und das Epithel ist stellenweise tiefer in die Wand eingedrungen. Auf einer Strecke von $1 \mathrm{~cm}$ ist das Bindegewebe strukturlos und sehr kernarm, und auch die Adventitia der Gefässe ist in der 
Nähe der Vagina in gleicher Weise verändert. Das Oberflächenepithel ist völlig normal. Der Darm enthält auf eine ungewöhnlich weite Strecke vom Anus aufwärts Plattenepithel, das selbst keine Ulzerationen zeigt. In dem subepithelialen Gewebe hingegen findet sich besonders in der Nähe des Uebergangs zum Zylinderepithel eine teilweise recht starke Leukozyteninfiltration, ebenso liegen in der Muskulatur und in dem Zwischengewebe stellenweise ziemlich zahlreiche Leukozyten, die in der Umgebung der Gefässe eine dichtere Ansammlung aufweisen. Die Muskulatur des Darmes zeigt ebenso wie das Gewebe der Scheidenwand keine Veränderungen. Während ich, wie gesagt, anfangs geneigt war, den Befund als eine Ausheilung von aussen her anzusehen, kamen uns später Bedenken, ob diese Deutung richtig sei. Herr Professor Mönckeberg, der so freundlich war, die Präparate durchzusehen. neigte ebenfalls zu der Ansicht, dass es sich hier wohl um einen atypischen Befund am Rektum und nicht um ein Ausheilungsbild handle. Immerhin muss man dann annehmen, dass in diesem Falle das Plattenepithel einen Schutz gegen die Zerstörung der Darmwand gebildet hat.

Wie dem auch sei, es haben jedenfalls die Befunde an Jen anderen Tieren eine überraschende Aehnlichkeit mit den Veränderungen beim Menschen gezeigt. Wir müssen darum auch nach diesen Befunden annehmen, dass die Geschwürs- und Fistelbildungen beim Menschen durch Ueberdosierung entstanden sind. Beides: die Tierexperimente und die klinischen Beobachtungen beim Menschen zeigen uns, wie ungleich empfindlicher die Darmschleimhant ist, die, obwohl sie weiter rom Bestrahlungsobjekt entfernt liegt, so viel früher schon recht weitgehende Veränderungen aufweisen kann. Das lässt uns auch verstehen, warum bei manchen Fraven zuweilen schon nach relativ kleinen Dosen so auffallend starke Darmerscheinungen auftreten können. Das deutet aber auch darauf hin, dass wir ähnliche Symptome seitens des Magens und Darmes, die wir nach abdominellen Röntgenbestrahlungen nicht so selten beobachten können, ebenfalls als ein Symptom der grösseren Schleimhautempfindlichkeit dieser Organe gegen die Röntgenstrahlen aufzufassen haben. 